coalition ou par toutes autres personnes (elles sont nombreuses) exigerait sûrement un autre découpage que celui que l'on connaît. Le prochain ouvrage sur la décentralisation devra se pencher sur cette nécessité, sinon, moi, je refuserai d'en faire la recension.

${ }^{1}$ Volume 16, No 1, hiver 2007

${ }^{2}$ Note de l'auteur.

${ }^{3}$ Volume 16, No 1, hiver 2007

${ }^{4}$ Volume 17 , no 1 , hiver 2008

\section{Pierre Merlin, L'aménagement $d u$ territoire en France, Paris, La Documentation française, 2007, 174 p.}

L'expression « aménagement du territoire (AduT) » se veut typiquement française même si les Québécois l'ont importée quelques années avant la création du Bureau d'aménagement de l'Est du Québec (le trop célèbre BAEQ dont il ne faut pas rappeler à la mémoire des Gaspésiens). Les Wallons refusent de l'employer et les Suisses, à ma connaissance, ne l'emploient guère. Reste, bien sûr, l'Afrique francophone qui ne se prive pas de l'utiliser à défaut d'aménager véritablement. L'expression a été popularisée avec la création de la Délégation à l'aménagement et à l'action régionale (DATAR) en 1963 avec Olivier Guichard, grand gaulliste devant l'éternel, qui en fut le tout premier délégué. Il s'agissait alors de conseiller l'État dans sa volonté de favoriser, à travers les fameux plans quinquennaux, un développement harmonieux de l'Hexagone en cherchant à décongestionner Paris et à favoriser un meilleur équilibre entre les régions. Amenuiser les disparités régionales, voilà l'idéal que reprendra à sa création, en 1969, notre cher Office de planification et de développement du Québec qui, en fait, ne planifiera guère tout en ne développant pas davantage. Mais ne dit-on pas que c'est l'intention qui compte? En ce qui regarde l'AduT, le concept faisait déjà partie des préoccupations du Conseil d'orientation économique, sous l'égide de Roland Parenteau, avant l'avènement de l'OPDQ. Le concept fut rapatrié à la faveur du retour de France de jeunes universitaires engagés sur les traces de leurs professeurs ayant obtenus leurs galons en France dans les années d'après guerre. Ces derniers avaient été recrutés par le père Lévesque, fondateur de la Faculté des sciences sociales au grand dam d'un certain premier ministre de l'époque car, contrairement aux évêques, lui, il refusait de manger dans sa main. En 1964, à titre de finissant d'une formation de premier cycle à l'Université Laval, l'AduT avait été le thème de mon séminaire de fin d'étude. À la bibliothèque, $\mathrm{j}$ 'avais pu trouver quatre ou cinq références... Cet ouvrage de
Pierre Merlin, professeur émérite de l'Université de Paris I, montre, ô combien, il s'en est coulé de l'eau sous le pont Neuf ou sous celui de Québec depuis lors. Car les références sur le sujet, aujourd'hui, comme les feuilles d'automne, se ramassent à la pelle ou presque.

L'auteur montre, en fait, que la notion d'AduT, a fait son apparition au lendemain de la guerre (la deuxième, bien sûr) alors qu'il fallait reconstruire l'Europe. Elle est devenue, comme il l'écrit, une priorité de l'action de l'État dans les années 1960 sous l'influence du volontariste gaulliste. Suite à une recension portant sur la décentralisation ${ }^{1}$, il est intéressant de voir comment en France s'est présenté le conflit centralisation versus décentralisation. Cette dernière remportera une bataille avec les lois Deferre 1982-83. Mais les partisans des Jacobins ne lâchant jamais prise, les disciples des Girondins ne sont jamais assurés de remporter une guerre qui n'en finit jamais de finir. L'évolution des différentes politiques françaises ne peut manquer d'intéresser tous ceux qui, au Québec, appellent de tous leurs vœux des réformes susceptibles de conduire à une diminution de l'emprise de Québec sur le sort des régions. Celui qui a écrit pas moins d'une cinquantaine d'ouvrages sur des sujets entourant la problématique de l'AduT, dégage un constat pessimiste en ce qui regarde la situation prévalant en France. À plusieurs titres, ose-til écrire, il est tenté de penser que les évolutions observées sur une période couvrant plus de quatre décennies, comme étant des régressions. Pas facile en effet, de décentraliser, surtout un pays comme la France dont le mal principal est justement le centralisme comme l'a signalé en son temps (1976) Alain Peyrefitte.

Mais de quoi parle-t-on vraiment? Aménager le territoire, pour l'auteur, c'est se rapporter à l'action et à la pratique de disposer avec ordre à travers l'espace d'un pays et dans une vision prospective, les hommes et leurs activités, les équipements et les moyens de communication qu'ils peuvent utiliser, en prenant en compte les contraintes naturelles, humaines et économiques, et ceci afin que les fonctions et les relations entre les hommes s'exercent de la façon la plus commode, la plus économique et la plus harmonieuse. Voilà, pas plus compliqué ${ }^{2} .$. Après s'être interrogé sì aménager un territoire relève d'une démarche scientifique, ou s'il ne s'agit pas plutôt d'une technique ou encore, comme le dit le Larousse, s'il s'agit d'une forme d'art, l'auteur préfère ramener l'AduT à son caractère d'intervention volontaire, de dimension territoriale de la planification. C'est donc une praxis, i.e. un ensemble d'actions visant un même objectif. Ceci étant admis, l'auteur s'interroge sur les fondements théoriques de l'AduT. Quel en est le contenu, doit-il être global ou peut-il être thématique? 
Comme les lecteurs de ma génération le devinent bien, l'historique de l'AduT ne peut manquer d'évoquer ici et là le fameux Paris ou le désert français du géographe J.F. Gravier. Ce pamphlet d'un jeune universitaire est apparu au lendemain de la guerre comme rien de moins qu'un gros pavé dans la mare. Oui, il fallait prendre conscience de la nécessité d'agir. L'auteur voir dans cette parution l'élément déclencheur de la première politique d'AduT qui sera suivi de beaucoup d'autres ici très bien décrites. En faisant un saut dans le temps, on en arrive à une section se rapportant à l'innovation et à la recherche. Il est donc question de technopole avec ou sans «^ ». L'auteur décèle ici l'influence de François Perroux en prenant l'exemple de Fos-sur-Mer, ce pôle qui en fait n'a jamais réalisé les espoirs fondés en lui avec l'avènement de la crise sidérurgique de la fin des années 1960. Un technopôle, précise l'auteur, réunit sur un site, en général suburbain, des activités faisant appel à des technologies innovantes et acceptées par un comité d'agrément. Il peut comporter un établissement d'enseignement supérieur ou un grand centre de recherche. Sofia-Antipolis se veut la première réalisation en France en étant crée de toute pièce en 1969 sur une initiative privée. Une sous-section traitant du développement local veut que cette notion ait été mise de l'avant dans les années 1990. En fait, c'est dans la décennie précédente qu'elle est apparue, soit avec l'arrivée du PS au pouvoir. Michel Rocard premierministre lors de la première mouture de l'administration Mitterrand s'en faisait le porte-étendard. La définition ici donnée mérite de se voir signalée : le développement local suppose une volonté collective de mobiliser les ressources locales, tant naturelles, qu'économiques et humaines, et de conserver la maîtrise de ce développement. Sur un territoire homogène, les acteurs locaux portent un projet global (à la fois social et économique) de développement. Devant souvent me plaindre qu'en France, l'expression développement local s'utilise à toutes les sauces, ce n'est heureusement pas le cas ici.

La section sur l'aménagement rural intéressera tous ceux qui prêtent attention à notre politique nationale de la ruralité. Ces lecteurs pourront faire le rapprochement entre nos pactes ruraux, qui en constituent l'épine dorsale, et l'expérience des contrats de pays initiés à la fin des années 1970 et surtout avec tout ce que recouvre l'expression «le retour des pays» à la faveur des différentes lois allant dans le sens de la décentralisation et adoptées en France au tournant du siècle. De même, la référence à l'expérience des parcs naturels régionaux est riche d'enseignements pour ceux qui en région, chez nous, travaillent à la mise en valeur de leur patrimoine naturel.

Quant aux urbains, surtout les Montréalais encore sous le choc de événements survenus à l'été 2008 à
Montréal-Nord, ils ne manqueront pas de trouver un grand intérêt dans la lecture de ce qui se rapporte aux banlieues et aux politiques de la ville. Une sous-section intitulée Grands ensembles et exclusion traite des quartiers les plus mal desservis ou règnent un taux de chômage élevé, un fort taux d'échec scolaire et la présence d'une forte proportion de gens d'origine étrangère. Cette dernière se trouve trop souvent marquée par l'exclusion qui sert de terreau à la violence, à la prolifération de la drogue, à l'insécurité et au racisme. Le phénomène des gangs de rue de Montréal trouve ici une explication particulièrement précise.

Enfin, faut-il voir à nouveau chez l'auteur un constat pessimiste lorsqu'il écrit qu'avec l'émergence des idées libérales (au sens européen du terme) et les contraintes de la mondialisation économique d'inspiration libérale, le concept d'AduT aurait perdu de sa force. Il voit dans la transformation de la DATAR en DIACT (Délégation interministérielle à l'aménagement et à la compétitivité des territoires) une conséquence de cette évolution. La compétitivité l'emportant sur l'aménagement, on donnera ainsi la priorité aux pôles de compétitivité qui font l'objet de la recension suivante.

\footnotetext{
${ }^{1}$ Voir l'ouvrage précédent.

${ }^{2}$ J'ai épargné le lecteur de parenthèses contenues dans cette définition.
}

Jean-Claude Némery, sous la dir. de, Les pôles de compétitivité dans le système français et européen : Approches sur les partenaires institutionnels, Paris, L'Harmattan, 2006, 197 p.

Il est étonnant de constater que le concept de pôle de compétitivité n'ait pas encore traversé l'océan pour être évoqué en relation avec le développement de nos régions comme il a été fait (à tort à mon avis) avec les concepts de district industriel ou de système de production local. Est-ce dû au fait, que sous l'administration du Parti québécois, au début des années 2000, un programme s'inspirant des mêmes prémisses fut mis de l'avant sous le nom d'Action concertée de coopération régionale de développement (ACCORD)? Ce programme, lancé dans une douzaine de régions du Québec, visait la réalisation d'un plan d'action économique régionale faisant appel aux entrepreneurs locaux et aux élites industrielles afin que chacun travaille de concert avec les sociétés d'État et les institutions financières. Un des postulats à la base de cette initiative voulait que le système productif régional regroupe l'ensemble des entreprises et des organisations 